\title{
Correction to: Patterns of prevalence and contemporary clinical management strategies in complicated acute biliary calculous disease: an ESTES 'snapshot audit' of practice
}

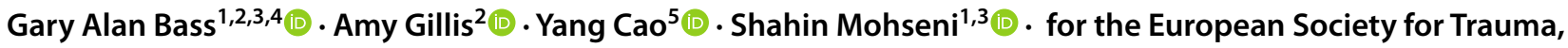 \\ Emergency Surgery (ESTES) Cohort Studies Group
}

Published online: 20 April 2021

(c) The Author(s) 2021

\section{Correction to: \\ European Journal of Trauma and Emergency Surgery https://doi.org/10.1007/s00068-020-01433-x}

The original version of this article unfortunately contained a copy-editing mistake. The collaborators of the "European Society for Trauma, Emergency Surgery (ESTES) Cohort Studies Group" were tagged incorrectly in the metadata.

The original article has been corrected.

The original article can be found online at https://doi.org/10.1007/ s00068-020-01433-x.

\section{Gary Alan Bass}

garybassmd@gmail.com

Amy Gillis

agillis@tcd.ie

Yang Cao

yang.cao@oru.se

Shahin Mohseni

shahin.mohseni@oru.se

1 Emergency Surgery Committee, European Society for Trauma and Emergency Surgery (ESTES), Pölten, Austria

2 Department of Surgery, Tallaght University Hospital, Dublin 24, Ireland

3 Department of Surgery, Örebro University School of Medical Sciences, Örebro, Sweden

4 Department of Traumatology, Surgical Critical Care and Emergency Surgery, University of Pennsylvania, Philadelphia, USA

5 Department of Clinical Epidemiology and Biostatistics, School of Medical Sciences, Örebro University, Örebro, Sweden
Acknowledgements Open access funding provided by Örebro University.

Members of the European Society for Trauma, Emergency Surgery (ESTES) Cohort Studies Group A. Shamiyeh, L. Rosetti, G. Klimbacher, B. Klugsberger (Kepler University Clinic, Linz, Austria); P. Healy, C. Moriarty, C. Power, N. Knightly, A. D. K. Hill (Beaumont Hospital, Dublin, Ireland); D. C. Winter, M. E. Kelly, B. E. Creavin, É. J. Ryan, C. C. Duffy (SVUH Institute for Clinical Outcomes Research and Education, Dublin, Ireland); M. Sugrue, M. H. Moore, L. Flanagan (Letterkenny University Hospital, Letterkenny, Ireland); J. Ryan, C. Keady, B. Fahey, K. L. McKevitt, K. Barry (Mayo University Hospital, Castlebar, Mayo, Ireland); K. C. Conlon, K. Mentor, A. KazemiNava, B. J. (Saint Vincent's University Hospital, Dublin, Ireland); P. F. 
Ridgway, D. O. Kavanagh, M. Whelan, M. Donnelly, C. McCarrick, U. Muhammad, T. M. Connelly, P. C. Neary (Tallaght University Hospital, Dublin, Ireland); S. Magalina, V. Cozza, A. LaGreca, D. Gui (Fondazione Policlinico Universitario ‘A. Gemelli’ IRCCS, Rome, Italy); A. Malagnino, M. Zago, M. Montuori (Policlinico San Pietro, Bergamo, Milan, Italy); A. Biloslavo, N. Samardzic, S. Fracon, D. Cosola, N. de Manzini (Clinica Chirurgica, Trieste University Hospital, Trieste, Italy); U. Fernandes, P. Avelar, R. Marques, A.S. Esteves, A. Marçal, C. Gomes (Centro Hospitalar de Trás-os-montes e Alto Douro, Vila Real, Portugal); D. Machado, T. Teles, S. Neves, M. Semiao, R. Cunha (Centro Hospitalar Cova de Biera, Covilha, Portugal); J. Pereira, J. Constantino, M. Sá, C. Casimiro (Centro Hospitalar Tondela-Viseu, Viseu, Portugal); L. Ionescu, R. Livadariu, L. Stirbu, R. Danila, D. Timofte, B. Astefaniei (St. Spiridon Emergency Universitary Hospital, Iasi, Romania); A. Landaluce Olavarria, B. Estraviz Mateos, J. Gonzalez Taranco, D. Gomez, J. Barrutia, J. Zeballos (Hospital Urduliz, Bizkaia, Spain); D. Morales Garcia, A. Lozano Najera, E. Gonzalez Tolaretxipi (University Hospital 'Marques de Valdecilla', Santander, Spain); L. Tallon-Aguilar, J. Pintor-Tortolero, A. Sanchez-Arteaga, V. Duran-Muñóz Cruzado, V. Camacho-Marente, J. Tinoco-Gonzalez (Hospital Virgen del Rocío, Seville, Spain); A. Älverdal (Jönköping Medical Center, Jönköping, Sweden); S. Redeen (Linköping University Hospital, Linköping, Sweden); S. Mohseni, A. Mohammad, R. Ahl, M. Wikström (Örebro University Hospital, Örebro, Sweden); S. Marinos,
N. Warner, R. Patel, T. Magro, R. Sunthareswaran (Stoke Mandeville Hospital, Aylesbury, UK); A. Mihailescu, G. Pokusewski, A. L. Bubuianu (Tameside General Hospital, Ashton-under-Lyme, UK); C. Dimitriu, M. Paraoan (Wrightington, Wigan and Leigh NHS Foundation Trust, Wigan, UK); A. Desai, K. Jones, M. Mlotshwa, K. Ross, S. Lambracos, Y. Tryliskyy (Worthing Hospital, Worthing, UK); D. C. Cullinane (Marshfield Clinic, Marshfield, Wisconsin, USA).

Open Access This article is licensed under a Creative Commons Attribution 4.0 International License, which permits use, sharing, adaptation, distribution and reproduction in any medium or format, as long as you give appropriate credit to the original author(s) and the source, provide a link to the Creative Commons licence, and indicate if changes were made. The images or other third party material in this article are included in the article's Creative Commons licence, unless indicated otherwise in a credit line to the material. If material is not included in the article's Creative Commons licence and your intended use is not permitted by statutory regulation or exceeds the permitted use, you will need to obtain permission directly from the copyright holder. To view a copy of this licence, visit http://creativecommons.org/licenses/by/4.0/. 